\title{
Do CVD grown graphene films have antibacterial activity on
}

metallic substrates?

L. Dellieu ${ }^{1, *,}$, E. Lawarée ${ }^{2, \S}$, N. Reckinger ${ }^{1,3 \S}$, C. Didembourg ${ }^{2}$, J.-J. Letesson ${ }^{2}$, M. Sarrazin $^{1,3}$, O. Deparis ${ }^{1}$, J.-Y. Matroule ${ }^{2, \$}$, and J.-F. Colomer ${ }^{1,3, \$}$

${ }^{1}$ Solid-State Physics laboratory, Department of Physics, University of Namur, Rue de Bruxelles 61, 5000 Namur, Belgium.

${ }^{2}$ Research Unit in Microorganisms Biology, University of Namur, Rue de Bruxelles 61, 5000 Namur, Belgium.

${ }^{3}$ Research group on carbon nanostructures (CARBONNAGe), University of Namur, Rue de Bruxelles 61, 5000 Namur, Belgium.

$\S, \$$ These authors contributed equally to this work.

Accurate assessment of the antibacterial activity of graphene requires consideration of both the graphene fabrication method and, for supported films, the properties of the substrate. Large-area graphene films produced by chemical vapor deposition were grown directly on copper substrates or transferred on a gold substrate and their effect on the viability and proliferation of the Gram-positive bacteria Staphylococcus aureus and the Gram-negative bacteria Escherichia coli were assessed. The viability and the proliferation of both bacterial species were not affected when they were grown on a graphene film entirely covering the gold substrate, indicating that conductivity plays no role on bacterial viability and graphene has no antibacterial activity against $S$. aureus and E. coli. On the other hand, antibacterial activity

"Corresponding author : Email address : louis.dellieu@unamur.be (L. Dellieu) FAX : +32 81724464 
was observed when graphene coated the copper substrates, resulting from the release of bactericidal cupric ions in inverse proportion to the graphene surface coverage.

\section{Introduction}

Graphene is a two-dimensional crystal composed of hybridized-sp ${ }^{2}$ carbon atoms in a hexagonal lattice structure that possesses many exceptional properties. Inter alia, it is a zerogap semiconductor, mechanically hard, extremely flexible, chemically inert, impermeable to any atom or molecule, and optically transparent [1]. Such properties allow the development of biomedical devices with the proviso that the observed or suspected interactions between biological systems and graphene are well understood. In this context, a few toxicity studies have recently been conducted on graphene, in particular regarding its antibacterial activity, which have led to conflicting results in some cases [2-8]. One of the main reasons of the controversy is the nature and the properties of the various graphene-based materials. Graphite, graphene oxide, reduced graphene oxide or chemical vapor deposition (CVD) produced graphene can lead to dramatically different results when their impact on biological systems is studied. Most of the studies have been carried out on graphene oxide [9-11], which requires a subsequent reduction step for converting it into graphene [12]. The proliferation of L-929 cells [2] or neuroendocrine PC12 cells [3] on graphene paper resulting from the reduction of graphene oxide suggests the biocompatibility of this material. On the contrary, other results have shown that, on such a paper, the membrane integrity of Escherichia coli (E. coli) DH5 strain was lost, which induced a bactericidal effect [4]. Graphene oxide nanowalls, because of their very sharp edges, damage cell envelopes during the contact interaction with both Gramnegative bacteria E. coli and Gram-positive bacteria Staphylococcus aureus (S. aureus) and exhibit an even stronger antibacterial activity when they are in a reduced form [5]. Recently, 
this phenomenon was explained by the penetration of graphene oxide nanowalls in the membranes of E. coli resulting in the extraction of phospholipids and thereby in a reduction of the bacterial viability [6]. The antibacterial activity towards E. coli was also shown to depend on the type of graphene materials, i.e. increased activity from graphite to reduced graphene oxide [7]. Conversely, Ruiz et al. demonstrated that graphene oxide supports E. coli growth and therefore does not possess antibacterial properties [8]. These conflicting conclusions most likely come from the structural and chemical characteristics of the studied graphene materials, mainly graphene oxide nanowalls, which have sharp edges and must undergo a reduction step involving the presence of chemical functions on their surfaces. The antibacterial activity of structurally flat graphene films, on other hand, is an interesting avenue to investigate.

Very recently, large-area graphene films produced by $\mathrm{CVD}$ on $\mathrm{Cu}$ or $\mathrm{Ge}$, or transferred to $\mathrm{SiO}_{2}$ were reported to possess an antibacterial activity that was thought to be related to the electronic properties of the substrate [13]. The antibacterial mechanism was hypothesized to involve electron transfer from the microbial membrane, via graphene, to the substrate which acts as an electron pump [13]. In the present study, we provide experimental evidence that rules out the electron transfer model as a mechanism explaining the presumed antibacterial activity of CVD graphene films. This calls for revisiting the problem in order to clarify the role of the substrate conductivity, keeping in mind that any study on antibacterial activity must meet the required standards in biology in order to be convincing [14]. For this purpose, we elaborated CVD graphene films on copper $(\mathrm{Cu})$ and gold $(\mathrm{Au})$ substrates. Either entirely or partially covering films were grown on $\mathrm{Cu}$ surfaces whereas entirely covering films were transferred on Au surfaces. The antibacterial activity of the different samples was tested on the Gram-positive bacteria S. aureus and the Gram-negative bacteria E. coli. 


\section{Experimental details}

\subsection{Graphene samples}

The graphene samples were grown by atmospheric pressure CVD on $\mathrm{Cu}$ foil pieces $[15,16]$ ( $\sim \mathrm{cm}^{2} ; 99.9 \%$ purity; $50-\mu \mathrm{m}$ thick) with dilute $\mathrm{CH}_{4}\left(95: 5 \mathrm{Ar}: \mathrm{CH}_{4}\right)$ as carbon precursor, using a hotwall furnace and a quartz reactor. The $\mathrm{Cu}$ foils were cleaned by sonication in acetone and isopropanol, and $\mathrm{Cu}$ was de-oxidized with acetic acid. The synthesis was next conducted at a temperature of $1050{ }^{\circ} \mathrm{C}$ for $1 \mathrm{~h}$ under flows of $\mathrm{Ar}(500 \mathrm{sccm}), \mathrm{H}_{2}(20 \mathrm{sccm})$, and dilute $\mathrm{CH}_{4}(0.2 \mathrm{sccm}$ for partial coverage and $1 \mathrm{sccm}$ for full coverage). After graphene growth, the quartz tube was extracted rapidly under flows of $\mathrm{Ar}$ and $\mathrm{H}_{2}$ in order to maintain the integrity of graphene [16]. Just after the synthesis, some $\mathrm{Cu}$ foils were covered by poly(methyl methacrylate) (PMMA) and etched by aqueous ammonium persulfate for subsequent transfer on $\mathrm{Au}$ substrates. After a few hours in the solution, the $\mathrm{Cu}$ foils were completely etched. The PMMA/graphene stacks were then copiously rinsed in distilled water in order to remove contaminants arising from the $\mathrm{Cu}$ etching step. Next, they were scooped from the solution with Au substrates and left to dry in air. After a second coating of PMMA [17], the samples were soaked in acetone for several hours to dissolve PMMA, rinsed in isopropyl alcohol and finally gently blown dry with nitrogen.

In order to verify the quality and the number of layers of the graphene films, they were transferred on $\mathrm{Si} / \mathrm{SiO}_{2}$ wafers and analyzed by Raman spectroscopy (see Fig. S1 in the supplementary information). Raman spectroscopy was performed at room temperature with a LabRam Horiba spectrometer at a laser wavelength of $514 \mathrm{~nm}$. Figure S1a shows optical microscope image of a graphene layer after transfer on a $\mathrm{Si} / \mathrm{SiO}_{2}$ piece. The main background layer is typically monolayer graphene (Raman spectrum in Fig. S1b: 2D peak position $=2693$ $\mathrm{cm}^{-1} ; \mathrm{G}$ peak position $=1590 \mathrm{~cm}^{-1} ; \mathrm{I}_{2 \mathrm{D}} / \mathrm{I}_{\mathrm{G}}=2.7$; full width at half maximum $\left.=33 \mathrm{~cm}^{-1}\right)$, with 
some bilayer islands (Raman spectrum in Fig. S1c: 2D peak position $=2713 \mathrm{~cm}^{-1}$; G peak position $=1592 \mathrm{~cm}^{-1} ; \mathrm{I}_{2 \mathrm{D}} / \mathrm{I}_{\mathrm{G}}=0.9$ ). In addition, the $2 \mathrm{D}$ peak of the bilayer graphene (full width at half maximum $=53 \mathrm{~cm}^{-1}$ ) can be fitted by four lorentzians (see Fig. S1c), thereby revealing that the analyzed bilayer graphene islands are AB-stacked. All these values are in good agreement with the literature [18,19] and our previous work [16]. The defect-related band (located at $\sim 1350 \mathrm{~cm}^{-1}$ ) is barely visible, testifying to the good quality of the transferred graphene films.

It is noteworthy that graphene oxide may lead to bactericidal effects [10]. In order to ensure that this effect can be excluded in our case, we have assessed the possibility of reactive oxygen species generation by the graphene sheets. We inspected graphene on $\mathrm{Cu}$ foils by $\mathrm{X}$ ray photoelectron spectroscopy (see reference [16] for more details) and found that the corresponding graphene film was not oxidized (within the detection limit of the equipment), as illustrated by C $1 s$ spectrum in the supplementary information Fig. S2 (in accordance with our previous results [16]). Therefore it is very unlikely that our graphene sheets generate reactive oxygen species.

\subsection{Bacterial live/dead analysis by flow cytometry}

After sterilization in a $75 \% \mathrm{v} / \mathrm{v}$ ethanol solution, triplicates of each surface $\left(1 \mathrm{~cm}^{2}\right)$ were placed in a 24-well plate (NuncTM). In order to reduce water evaporation from the bacterial solution, the remaining wells and the empty spaces between them were filled with deionized water. Sixty $\mu 1$ of a bacterial suspension containing $6 \times 10^{6} \mathrm{CFU} / \mathrm{ml}$ of $S$. aureus (ATCC 25923 ) or $2 \times 10^{7} \mathrm{CFU} / \mathrm{ml}$ of E. coli (ATCC 25922) were poured onto the surfaces and incubated for $24 \mathrm{~h}$ at $37^{\circ} \mathrm{C}$. In order to assess bacterial viability, bacteria were diluted in a

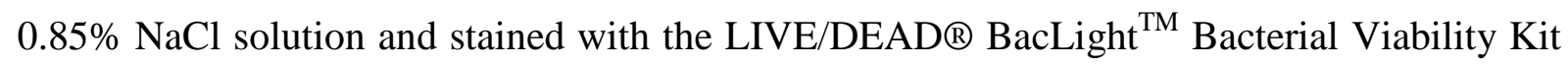
(Molecular Probes ${ }^{\circledR}$ ) according to the manufacturer protocol. 50,000 events per experimental 
condition were acquired with a BD FACSCalibur ${ }^{\mathrm{TM}}$ flow cytometer equipped with an argon laser at $488 \mathrm{~nm}$. Data acquisition and analysis were performed by using the BD CellquestPro $^{\mathrm{TM}}$ software. The bacterial population was distributed in two regions of the logintegrated red (Propidium iodide:FL3) versus green (Syto9:FL1) plot and the number of bacteria found in these regions were used to estimate the percentage of bacterial viability. The non-bacterial events were eliminated by gating the SSC/FSC (granularity against cell size) plot. To ensure the statistical validity of the results, each experiment was repeated three times (biological replicate) and each biological replicate was analyzed three times (technical replicate).

\subsection{Measurement of $\mathrm{Cu}$ and $\mathrm{Au}$ concentration by atomic absorption} spectroscopy

The metallic ions concentration in the bacterial suspensions was measured by using atomic absorption spectroscopy (AAS) (AA-7000F from Shimadzu). The AAS was calibrated with different standard solutions of the analysed element. Bacterial suspensions were diluted 100 times in $1 \mathrm{M} \mathrm{HNO}_{3}$. The $\mathrm{Cu}$ or $\mathrm{Au}$ absorbance of each biological replicate was measured three times as technical replicates.

\subsection{Evaluation of the antibacterial activity}

The bacterial suspension, incubated for $24 \mathrm{~h}$ on the surfaces described above, was collected and serially diluted in liquid LB medium. Twenty $\mu$ l of each dilution were inoculated on LB agar plates. After an overnight incubation at $37^{\circ} \mathrm{C}$, the culture plates were photographed and the total bacterial charge was estimated by counting the Colony Forming Units (CFU). To ensure the statistical validity of the results, the experiment was repeated three times 
(biological triplicate). Each biological replicate was inoculated on three different LB agar plates (technical replicate).

\subsection{Scanning electron microscopy analysis}

Scanning electron microscopy (SEM) was performed using a JEOL 7500 F microscope under an accelerating voltage of $1 \mathrm{kV}$, with a working distance of $3 \mathrm{~mm}$, and an emission current of $5 \mu \mathrm{A}$. The samples were not coated with any metallic thin layer.

\section{Results and discussion}

\subsection{Production and characterization of the graphene/metal samples}

The use of a non-biologically inert metallic substrate, such as $\mathrm{Cu}$, which was previously used to test graphene antibacterial activity [13] is expected to bias the assessment of this activity considering the bactericidal and bacteriostatic properties of $\mathrm{Cu}[20]$. As opposed to $\mathrm{Cu}, \mathrm{Au}$ is barely toxic for bacterial or animal cells due to its elemental properties [21]. Therefore, Au is an excellent candidate to test the antibacterial activity of graphene films on metallic substrates and to assess the contribution of the substrate conductivity. Both substrates have been considered in the present study.

As already mentioned here above, the graphene films were synthesized by atmospheric pressure $\mathrm{CVD}$ on $\mathrm{Cu}$ foils $[15,16]$ to produce two types of samples. The first one, named graphene@ $\mathrm{Cu}$, entirely covers the $\mathrm{Cu}$ surface and is composed of micrometer-sized domains of predominantly monolayer graphene with some bi- and trilayer graphene islands. SEM images (Fig. 1a-b) show a graphene film entirely covering the $\mathrm{Cu}$ substrate. The low magnification SEM image (Fig. 1a) is poorly contrasted because graphene completely overlays the $\mathrm{Cu}$ surface. In Fig. 1b, areas with different contrasts are seen: the darker the contrast, the greater the number of layers. Wrinkles, folds in graphene due to the difference 
between the thermal expansion coefficients of $\mathrm{Cu}$ and graphene, are also seen to cross $\mathrm{Cu}$ grain boundaries (Insert to Fig. 1b), testimony to the continuity of the graphene film [22].

The second type of sample, named graphene ${ }^{+/} @ \mathrm{Cu}$, is composed of single hexagonal domains that merge to form larger ones. In stark contrast with Fig. 1a-b, the low magnification SEM image (Fig. 1c) reveals a strong contrast between $\mathrm{Cu}$ and graphene because graphene hexagonal domains, about one to two tens of microns in size, do not fully cover the $\mathrm{Cu}$ foil. Moreover, these domains are not only composed of graphene monolayer but also occasionally of bi- or trilayer flakes (Fig. 1d). Graphene surface coverage was estimated to be around $80 \%$ from SEM image analysis.

Another type of sample, named graphene@Au, consists of continuous films of graphene deposited on Au substrates by transfer. Graphene was obtained exactly in the same CVD synthesis conditions as for graphene@ $\mathrm{Cu}$ and was transferred on Au substrates by using the polymer-assisted technique [23]. Finally, bare $\mathrm{Cu}$ and $\mathrm{Au}$ substrates are used as control samples.

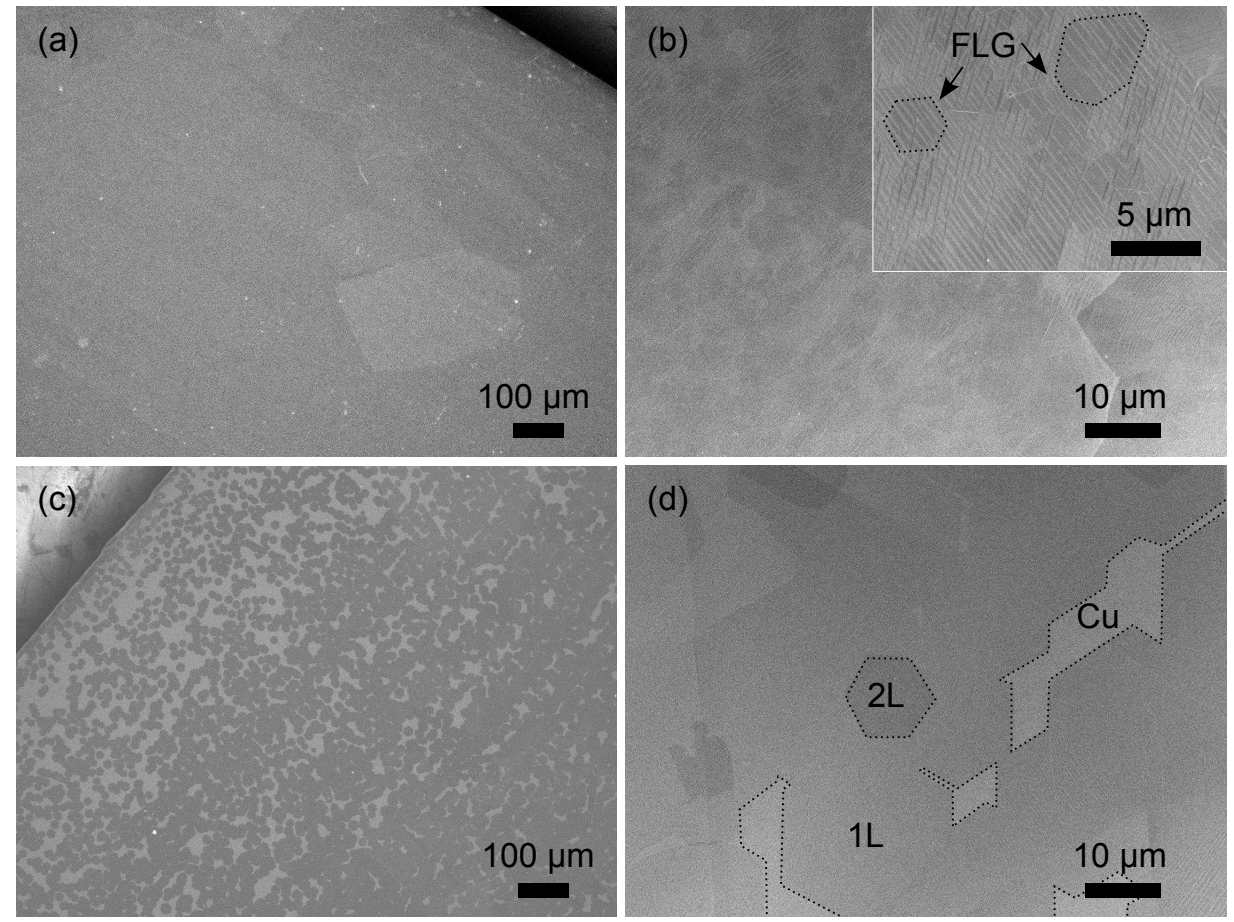


Fig 1: Morphology of graphene on Cu. (a) Low magnification SEM image of graphene@Cu showing the complete coverage of graphene on the $\mathrm{Cu}$ surface. (b) Medium magnification SEM image of graphene@Cu. Insert: Higher magnification SEM image where few-layer graphene domains are highlighted. The contrast differences are due to graphene domains with different numbers of layers, $\mathrm{Cu}$ grain orientations and boundaries, or graphene wrinkles. (c) Low magnification SEM image of graphene ${ }^{+/-} @ \mathrm{Cu}$ showing graphene hexagonal domains (dark grey islands) on the $\mathrm{Cu}$ substrate (light grey background), evidencing the partial coverage of the $\mathrm{Cu}$ surface. (d) Medium magnification SEM image of graphene ${ }^{+/-} @ \mathrm{Cu}$ showing the contrast difference between the graphene domains and the $\mathrm{Cu}$ substrate, and also between different numbers of graphene layers (1L: monolayer, 2L: bilayer).

\subsection{Graphene has no antibacterial activity.}

The bacterial envelope is a finely regulated physical barrier between the intracellular and extracellular environments. Severe structural alterations of this barrier are usually nonreversible and lead to bacterial cell death. Graphene bactericidal properties were assessed in the Gram-positive $S$. aureus and the Gram-negative E. coli with the so called "LIVE/DEAD" assay which relies on the level of bacterial envelope integrity to discriminate live from dead bacteria. Combination of the LIVE/DEAD assay with flow cytometry provides a quantitative insight of bacterial cell death (see Fig. S3 in supplementary information). S. aureus or E. coli cultures were seeded into a 24-well plate containing (i) bare $\mathrm{Cu}$ or bare $\mathrm{Au}$, (ii) a $\mathrm{Cu}$ foil partially (graphene ${ }^{+/-} @ \mathrm{Cu}$ ) or fully covered (graphene@ $\mathrm{Cu}$ ) with graphene or (iii) an Au foil fully covered with graphene (graphene@Au). After a $24 \mathrm{~h}$ incubation at $37{ }^{\circ} \mathrm{C}$, the cell cultures were recovered and subjected to the LIVE/DEAD assay. Interestingly, S. aureus and E. coli grown on graphene@Cu or graphene@Au exhibit at least 91\% viability relative to the 
$97 \%$ and $94 \%$ viability of the control S. aureus and E. coli cultures grown directly on the bottom of the 24-well plate (Fig. 2 a-b). Statistical analysis reveals that the difference between graphene@ $\mathrm{Cu}$ condition (but not the graphene@ $\mathrm{Au}$ condition) and the negative control is significant (p-value of 0.0004 for $S$. aureus, p-value of 0.0106 for E. coli). Accordingly, only $66 \%$ of $S$. aureus and $54 \%$ of $E$. coli could survive when grown on graphene ${ }^{+/} @ \mathrm{Cu} . \quad S$. aureus cells grown on bare $\mathrm{Cu}$ all died whereas $6 \%$ of $E$. coli cells survived under the same conditions (Fig. 2 a-b). AAS performed on the $S$. aureus and E. coli culture medium after the $24 \mathrm{~h}$ incubation revealed $\mathrm{a} \sim 19 \mathrm{mM}, \sim 6 \mathrm{mM}$ and $\sim 0.2 \mathrm{mM} \mathrm{Cu}$ concentration for bare $\mathrm{Cu}$, graphene $^{+/-} @ \mathrm{Cu}$ and graphene@ $\mathrm{Cu}$, respectively (Fig $2 \mathrm{c}-\mathrm{d}$ ), demonstrating the release of cupric ions into the culture medium and, therefore, the relative permeability of the graphene sheets. Indeed, graphene was previously shown to be an imperfect barrier owing to the presence of defects and domain boundaries [24,25]. Au concentration in the culture medium remained very low $(\sim 0.8 \mu \mathrm{M})$ under any experimental conditions, likely due to its low solubility.

Together, these results suggest that graphene per se has no bactericidal activity. Nevertheless, one cannot rule out any bacteriostatic effect of graphene films, where S. aureus and E. coli cells would survive to graphene in a non-replicative form. In order to test this hypothesis, we performed a CFU assay which quantitatively measures the ability of bacteria to divide and to give macroscopic colonies (see Fig. S4 in supplementary information) [26]. Twenty-four hours $S$. aureus and E. coli cultures performed under the experimental conditions described above were serially diluted and seeded on agar culture plates which were further incubated overnight until CFUs counting. 

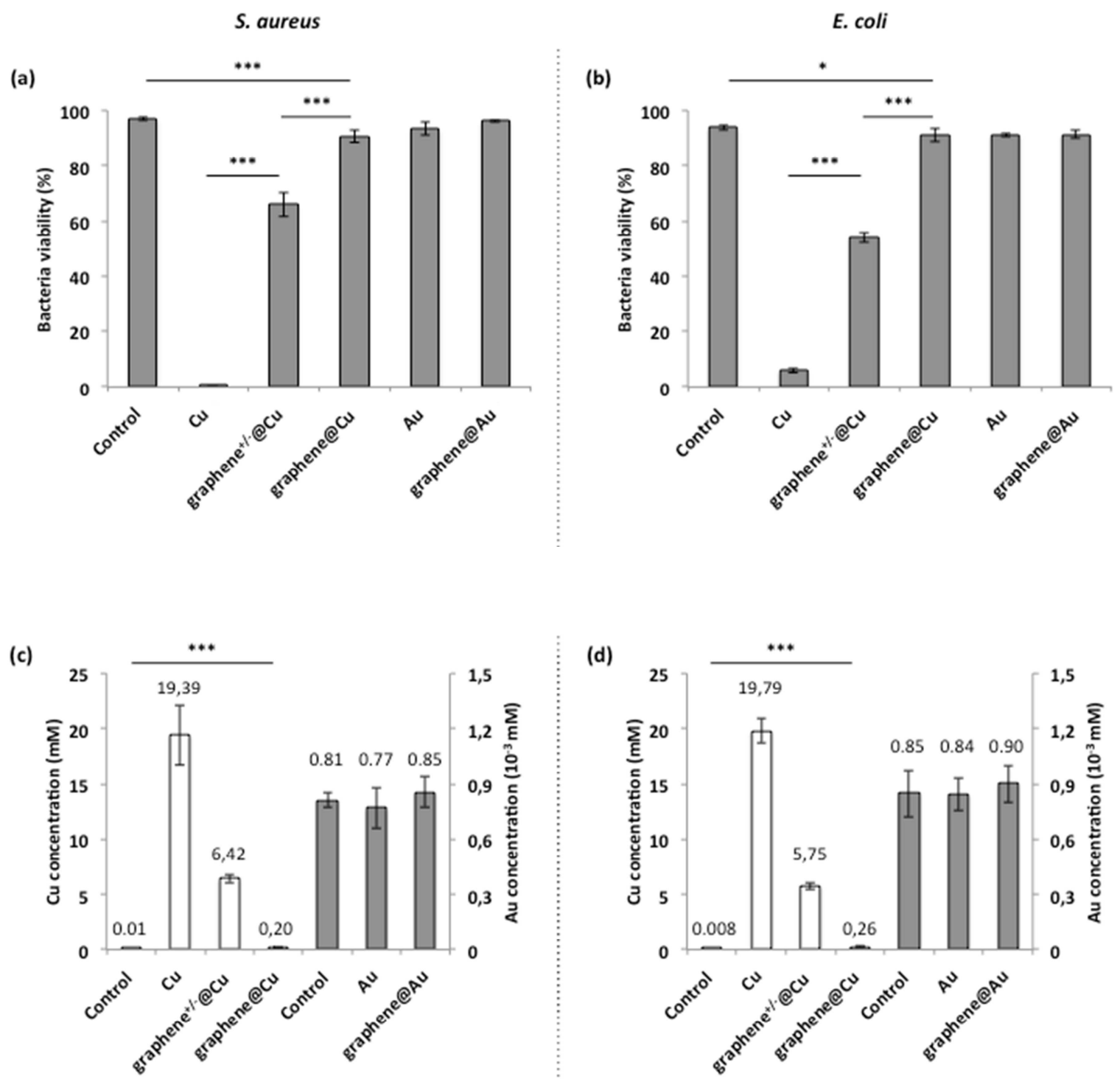

Figure 2: Graphene per se has no impact on $S$. aureus and $E$. coli viability. Bacterial viability was determined using the LIVE/DEAD assay after a $24 \mathrm{~h}$ incubation of $S$. aureus (a) and E. coli $(\mathrm{b})$ on bare $\mathrm{Cu}$, bare $\mathrm{Au}$,graphene ${ }^{+-} @ \mathrm{Cu}$, graphene@ $\mathrm{Cu}$ and graphene@ $\mathrm{Au} . \mathrm{Cu}$ and Au concentrations were measured by AAS after incubating S. aureus (c) and E. coli (d) for $24 \mathrm{~h}$ under the same experimental conditions. Stars denote the statistical significance (pvalues) in increasing order: $\mathrm{p}<0.1(*), \mathrm{p}<0.05(* *), \mathrm{p}<0.01(* * *)$. Error bars indicate standard deviation values. 
During the $24 \mathrm{~h}$ incubation in the 24-well plate, the control S. aureus and E. coli cultures underwent a 70000 and 700 fold increase of the CFU/ml, respectively, indicating that both bacterial species are able to proliferate under these conditions (Fig. 3 a-b). A similar pattern of proliferation was observed when both $S$. aureus and E. coli were grown on graphene@Au corroborating the lack of cytotoxicity of the graphene@Au evidenced by the LIVE/DEAD assay. When grown on graphene ${ }^{+/-} @ \mathrm{Cu}$ or graphene@ $\mathrm{Cu}$, both S. aureus and E. coli cells displayed a decrease of their proliferation rate, likely due to the toxicity of the released cupric ions. Logically, no CFU was observed when $S$. aureus was grown on bare $\mathrm{Cu}$, which did not completely abolish the capacity of $E$. coli to proliferate though since $0.01 \%$ of the seeded $E$. coli cells could give rise to a colony. These $0.01 \%$ are still much below the $6 \%$ survival measured with the LIVE/DEAD assay (Fig. 2 b), suggesting a bacteriostatic effect of the released cupric ions on E. coli. We therefore conclude that graphene has no significant intrinsic antibacterial activity when it is deposited on a biologically inert metallic substrate.

\subsection{Discussion}

Our results demonstrate that graphene, as far as it is produced by CVD and deposited on a conductive (metallic) substrate, does not display any significant antibacterial property against S. aureus and E. coli. Our study refutes the electron transfer model recently proposed by Li et al. to explain the antibacterial activity of large-area graphene films coated on $\mathrm{Cu}$ [13]. From a physical point of view, Li et al.'s model is questionable because it neglects the fact that graphene/metal junction properties must be carefully considered when estimating work 
S. aureus

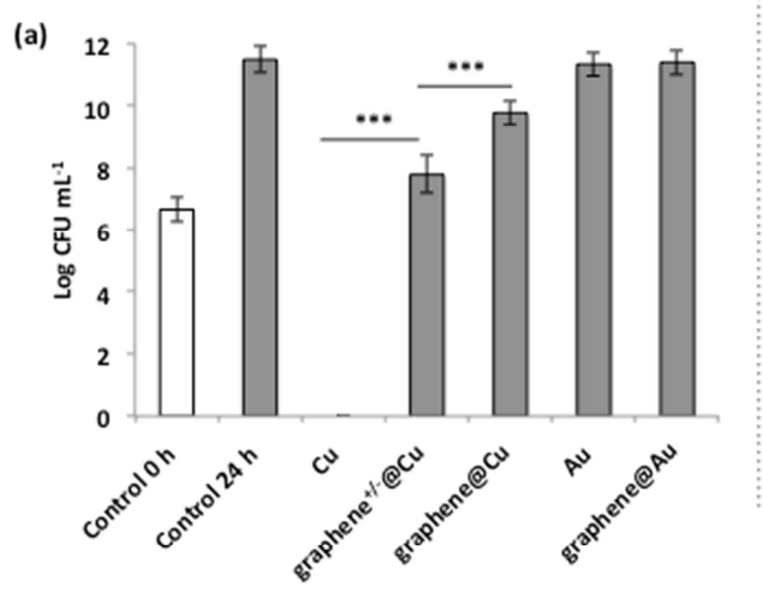

E. coli

(b)

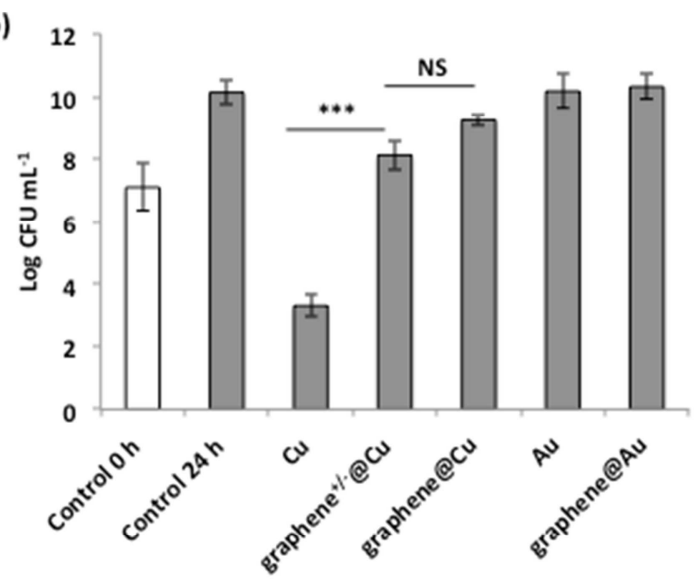

Fig. 3: Graphene per se has no antibacterial activity on $S$. aureus and $E$. coli. The ability of S. aureus (a) and E. coli (b) to proliferate upon a $24 \mathrm{~h}$ incubation (black bars) on the plastic bottom (control $24 \mathrm{~h}$ ), on bare $\mathrm{Cu}$, bare $\mathrm{Au}$, graphene ${ }^{+/} @ \mathrm{Cu}$, graphene@ $\mathrm{Cu}$ and graphene@Au was assessed by counting the CFU obtained after serially diluting and overnight plating the bacteria on solid medium. One control bacteria sample (control $0 \mathrm{~h}$ ) was also immediately subjected to CFU counting with no $24 \mathrm{~h}$ incubation (white bar) in order to determine the proliferation of the control bacteria. Stars denote the statistical significance (pvalues) in increasing order: $\mathrm{p}<0.1(*), \mathrm{p}<0.05(* *), \mathrm{p}<0.01(* * *)$. Error bars indicate standard deviation values. NS : non significant.

functions of materials [27-34]. First, when graphene rests on $\mathrm{Cu}$, it is known that it should be usually $n$-doped [27-31]. That means that electrons tend to be transferred from $\mathrm{Cu}$ to graphene, and not the opposite way. Consequently, graphene@ $\mathrm{Cu}$ should hardly allow electron transfer from graphene to $\mathrm{Cu}$ unlike what is assumed in $\mathrm{Li}$ et al.'s model. In fact, considering the theoretical current-voltage characteristic of graphene@ $\mathrm{Cu}$, the result predicted by Yamacli [29] is just the opposite of the one expected by Li et al.'s assumption. By 
contrast, graphene@Au is p-doped [29-34] and therefore electrons tend to be transferred from graphene to $\mathrm{Au}$. In this case, the theoretical current-voltage characteristic of graphene@ $\mathrm{Au}$ [29] meets the assumption of Li et al.'s model in terms of electron transfer direction. Now, as a matter of fact, no antibacterial activity was observed experimentally with graphene@ $\mathrm{Au}$ although electron transfer from graphene to $\mathrm{Au}$ is well known to occur efficiently [29-34]. Therefore, we can conclude that the proposed electron transfer mechanism is not pertinent as far as the interaction between bacteria and graphene on conductive substrates is concerned.

The electron transfer model is also highly questionable from a biological point of view because, according to the $\mathrm{Li}$ et al., it involves an electron transfer from the bacterial respiratory chain to an external electron acceptor through an "electron conduit". Respiration in bacteria is an energy generating process that relies on electron transport using a chain of proteins located in the cytoplasmic membrane to a terminal electron acceptor. This transport, coupled to the extrusion of protons $\left(\mathrm{H}^{+}\right)$, leads to a charge and $\mathrm{pH}$ gradient through the membrane. These charge carriers are far from being in contact with the external medium. In most cases, this does neither involves an extra-cellular electron acceptor nor a bacterial nanowire as claimed in Li et al.'s article. With regard to S. aureus, in aerobic conditions, the respiratory chain uses molecular $\mathrm{O}_{2}$ as a final acceptor and this gas freely diffuse to the cytoplasmic membrane [35]. According to our results, the hypothesis of facile transfer of electrons from microbial membranes to graphene does not seem satisfactory at all. Indeed, we do not observe any antibacterial effect with graphene@Au surfaces, despite that the Augraphene junction should favor such a hypothetical transfer. Notwithstanding that the $\mathrm{Cu}$ graphene junction should not facilitate electron transfer, we observe here an antibacterial activity due to released cupric ions which is in inverse proportion to graphene surface coverage. Indeed, the bactericidal property of cupric ions must be taken into account since the dissolved cupric ions cause membrane damage and cell disintegration [37]. 


\section{Conclusion.}

The absence of antibacterial activity of large-area CVD graphene films on conductive ( $\mathrm{Au}$, $\mathrm{Cu}$ ) substrates was demonstrated for $S$. aureus and $E$. coli. This result implies that the conductive character of the substrate has no influence on the viability of S. aureus and E. coli bacteria in contact with CVD graphene films. When a $\mathrm{Cu}$ substrate is used, however, the release of cupric ions from areas not covered by the graphene film led to an antibacterial effect which depends on the degree of graphene coverage. This role of the $\mathrm{Cu}$ substrate should be carefully taken into account in any assessment study on graphene antibacterial activity.

\section{References}

[1] Novoselov KS, Fal'ko VI, Colombo L, Gellert PR, Schwab MG, Kim K. A roadmap for graphene. Nature 2012;490:192-200.

[2] Chen H, Müller MB, Gilmore KJ, Wallace GG, Li D. Mechanically Strong, Electrically Conductive, and Biocompatible Graphene Paper. Adv Mater 2008;20:3557-61.

[3] Agarwal S, Zhou X, Ye F, He Q, Chen GCK, Soo J, et al. Interfacing Live Cells with Nanocarbon Substrates. Langmuir 2010;26:2244-7.

[4] Hu W, Peng C, Luo W, Lv M, Li X, Li D, et al. Graphene-Based Antibacterial Paper. ACS Nano 2010;4:4317-23.

[5] Akhavan O, Ghaderi E. Toxicity of Graphene and Graphene Oxide Nanowalls Against Bacteria. ACS Nano 2010;4:5731-6. 
[6] Tu Y, Lv M, Xiu P, Huynh T, Zhang M, Castelli M, et al. Destructive extraction of phospholipids from Escherichia coli membranes by graphene nanosheets. Nat Nano 2013;8:594-601.

[7] Liu S, Zeng TH, Hofmann M, Burcombe E, Wei J, Jiang R, et al. Antibacterial Activity of Graphite, Graphite Oxide, Graphene Oxide, and Reduced Graphene Oxide: Membrane and Oxidative Stress. ACS Nano 2011;5:6971-80.

[8] Ruiz ON, Fernando KAS, Wang B, Brown NA, Luo PG, McNamara ND, et al. Graphene Oxide: A Nonspecific Enhancer of Cellular Growth. ACS Nano 2011;5:8100-7.

[9] Stankovich S, Dikin DA, Dommett GHB, Kohlhaas KM, Zimney EJ, Stach EA, et al. Graphene-based composite materials. Nature 2006;442:282-6.

[10] Akhavan O, Ghaderi E. Escherichia coli bacteria reduce graphene oxide to bactericidal graphene in a self-limiting manner. Carbon 2012;50:1853-60.

[11] Akhavan O, Ghaderi E, Esfandiar A. Wrapping bacteria by graphene nanosheets for isolation from environment, reactivation by sonication, and inactivation by near-infrared irradiation. J Phys Chem B 2011;115:6279-88.

[12] Stankovich S, Dikin DA, Piner RD, Kohlhaas KA, Kleinhammes A, Jia Y, et al. Synthesis of graphene-based nanosheets via chemical reduction of exfoliated graphite oxide. Carbon 2007;45:1558-65.

[13] Li J, Wang G, Zhu H, Zhang M, Zheng X, Di Z, et al. Antibacterial activity of large-area monolayer graphene film manipulated by charge transfer. Sci Rep 2014;4.

[14] Kostarelos K, Novoselov KS. Exploring the Interface of Graphene and Biology. Science 2014;344:261-3. 
[15] Vlassiouk I, Regmi M, Fulvio P, Dai S, Datskos P, Eres G, et al. Role of Hydrogen in Chemical Vapor Deposition Growth of Large Single-Crystal Graphene. ACS Nano 2011;5:6069-76.

[16] Reckinger N, Felten A, Santos CN, Hackens B, Colomer J-F. The influence of residual oxidizing impurities on the synthesis of graphene by atmospheric pressure chemical vapor deposition. Carbon 2013;63:84-91.

[17] Li X, Zhu Y, Cai W, Borysiak M, Han B, Chen D, et al. Transfer of Large-Area Graphene Films for High-Performance Transparent Conductive Electrodes. Nano Lett 2009;9:4359-63.

[18] Ferrari AC, Meyer JC, Scardaci V, Casiraghi C, Lazzeri M, Mauri F, et al. Raman spectrum of graphene and graphene layers. Phys Rev Lett 2006;97: 187401.

[19] Malard LM, Pimenta MA, Dresselhaus G, Dresselhaus MS. Raman spectroscopy in graphene. Phys Rep 2009;473:51-87.

[20] Noyce JO, Michels H, Keevil CW. Potential use of copper surfaces to reduce survival of epidemic meticillin-resistant Staphylococcus aureus in the healthcare environment. J Hosp Infect 2006;63:289-97.

[21] Webster, T. J. Safety of Nanoparticles. Springer 2008.

[22] Li X, Cai W, An J, Kim S, Nah J, Yang D, et al. Large-Area Synthesis of High-Quality and Uniform Graphene Films on Copper Foils. Science 2009;324:1312-4.

[23] Reina A, Jia X, Ho J, Nezich D, Son H, Bulovic V, et al. Large Area, Few-Layer Graphene Films on Arbitrary Substrates by Chemical Vapor Deposition. Nano Lett 2009;9:30-5. 
[24] Zhou F, Li Z, Shenoy GJ, Li L, Liu H. Enhanced Room-Temperature Corrosion of Copper in the Presence of Graphene. ACS Nano 2013;7:6939-47.

[25] Schriver M, Regan W, Gannett WJ, Zaniewski AM, Crommie MF, Zettl A. Graphene as a Long-Term Metal Oxidation Barrier: Worse Than Nothing. ACS Nano 2013;7:5763-8.

[26] Kell DB, Kaprelyants AS, Weichart DH, Harwood CR, Barer MR. Viability and activity in readily culturable bacteria: a review and discussion of the practical issues. Antonie Van Leeuwenhoek 1998;73:169-87.

[27] Frank O, Vejpravova J, Holy V, Kavan L, Kalbac M. Interaction between graphene and copper substrate: The role of lattice orientation. Carbon 2014;68:440-51.

[28] Marsden AJ, Asensio M-C, Avila J, Dudin P, Barinov A, Moras P, et al. Is graphene on copper doped? Phys Status Solidi RRL 2013;7:643-6.

[29] Yamacli S. First principles study of the voltage-dependent conductance properties of ntype and p-type graphene-metal contacts. Computational Materials Science 2014;81:607-11.

[30] Nouchi R, Saito T, Tanigaki K. Determination of Carrier Type Doped from Metal Contacts to Graphene by Channel-Length-Dependent Shift of Charge Neutrality Points. Appl Phys Express 2011;4:035101.

[31] Giovannetti G, Khomyakov PA, Brocks G, Karpan VM, van den Brink J, Kelly PJ. Doping Graphene with Metal Contacts. Phys Rev Lett 2008;101:026803.

[32] Ma J, Xie G, Lv P, Gao W, Yuan P, Qian L, et al. Wavelength-Versatile Graphene-Gold Film Saturable Absorber Mirror for Ultra-Broadband Mode-Locking of Bulk Lasers. Sci Rep $2014 ; 4$. 
[33] Sławińska J, Dabrowski P, Zasada I. Doping of graphene by a Au(111) substrate: Calculation strategy within the local density approximation and a semiempirical van der Waals approach. Phys Rev B 2011;83:245429.

[34] Malec CE, Davidović D. Electronic properties of Au-graphene contacts. Phys Rev B 2011;84:033407.

[35] Hammer ND, Reniere ML, Cassat JE, Zhang Y, Hirsch AO, Indriati Hood M, et al. Two heme-dependent terminal oxidases power Staphylococcus aureus organ-specific colonization of the vertebrate host. MBio 2013;4.

[36] Santo CE, Lam EW, Elowsky CG, Quaranta D, Domaille DW, Chang CJ, et al. Bacterial Killing by Dry Metallic Copper Surfaces. Appl Environ Microbiol 2011;77:794-802.

\section{Acknowledgments.}

A part of this work is financially supported by the Belgian Fund for Scientific Research (F.R.S.-FNRS) under FRFC contract "Chemographene”' (No. 2.4577.11). L. D. and E. L. are supported by the Belgian Fund for Industrial and Agricultural Research (FRIA). J.-F. Colomer is supported by the F.R.S.-FNRS Research Associate. This research used resources of the Electron Microscopy Service located at the University of Namur ("Plateforme Technologique Morphologie - Imagerie"). The authors thank Carole Morel, Pierre Cambier and Eloise Van Hooijdonk for their technical support and advice. The authors acknowledge Xiaohui Tang and Benoît Hackens for their help with Raman measurements. 


\section{Supplementary information}

\section{Do CVD grown graphene films have antibacterial activity on}

metallic substrates?

L. Dellieu ${ }^{2, *,}$, E. Lawarée ${ }^{2, \S}$, N. Reckinger ${ }^{1,3 \S}$, C. Didembourg ${ }^{2}$, J.-J. Letesson ${ }^{2}$, M. Sarrazin $^{1,3}$, O. Deparis ${ }^{1}$, J.-Y. Matroule ${ }^{2, \$}$, and J.-F. Colomer ${ }^{1,3, \$}$

${ }^{1}$ Solid-State Physics laboratory, Department of Physics, University of Namur, Rue de Bruxelles 61, 5000 Namur, Belgium.

${ }^{2}$ Research Unit in Microorganisms Biology, University of Namur, Rue de Bruxelles 61, 5000 Namur, Belgium.

${ }^{3}$ Research group on carbon nanostructures (CARBONNAGe), University of Namur, Rue de Bruxelles 61, 5000 Namur, Belgium.

$\$$ \$ These authors contributed equally to this work. 

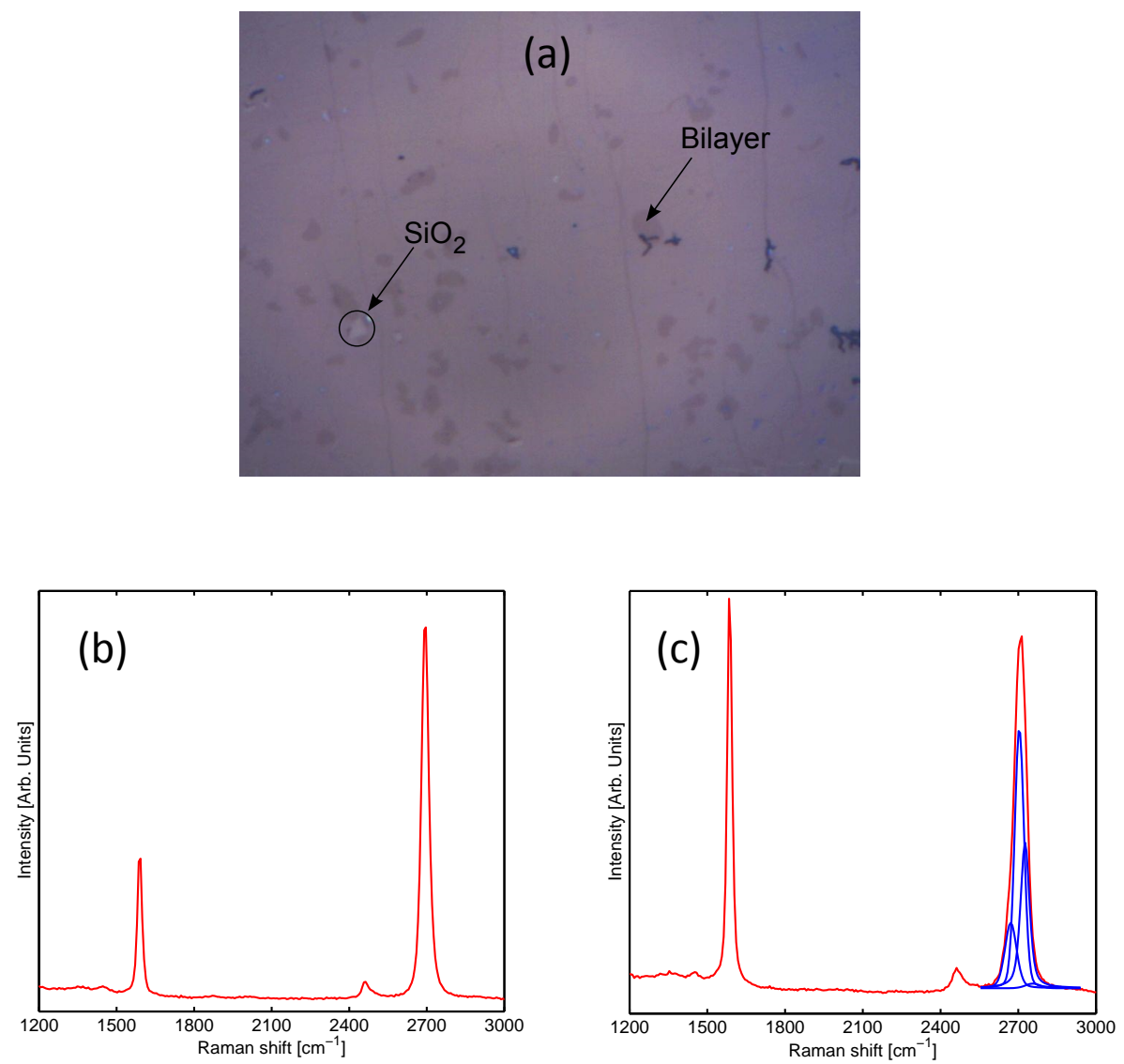

Fig. S1 - (a) Optical image of a graphene film transferred onto a $\mathrm{Si} / \mathrm{SiO}_{2}$ substrate. Corresponding Raman spectra for (b) monolayer and (c) bilayer graphene, respectively. The 2D peak of bilayer graphene can be fitted with 4 lorentzians, indicating that it is AB-stacked. 


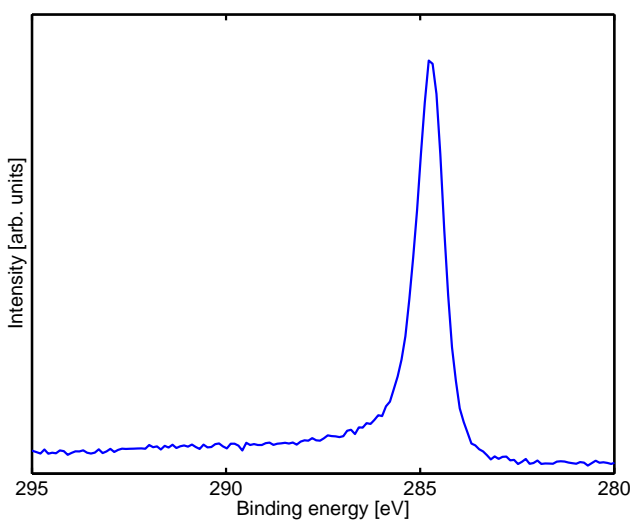

Fig. S2 - C $1 s$ X-ray photoelectron spectroscopy spectrum of graphene grown on copper foils, showing that graphene is not oxidized. 
S. aureus

(a)

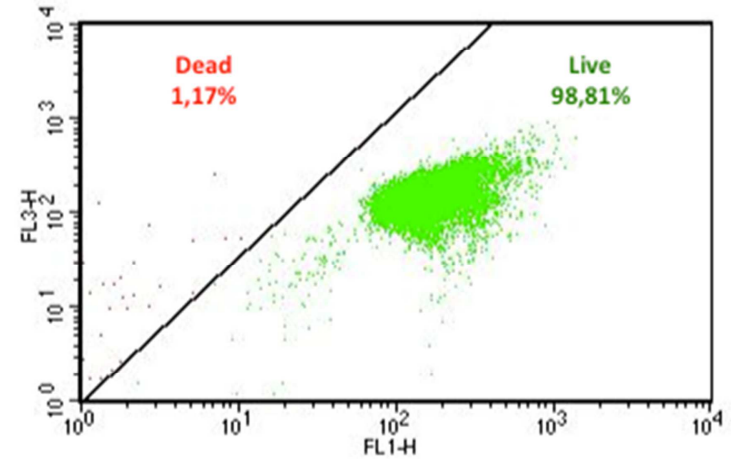

(c)

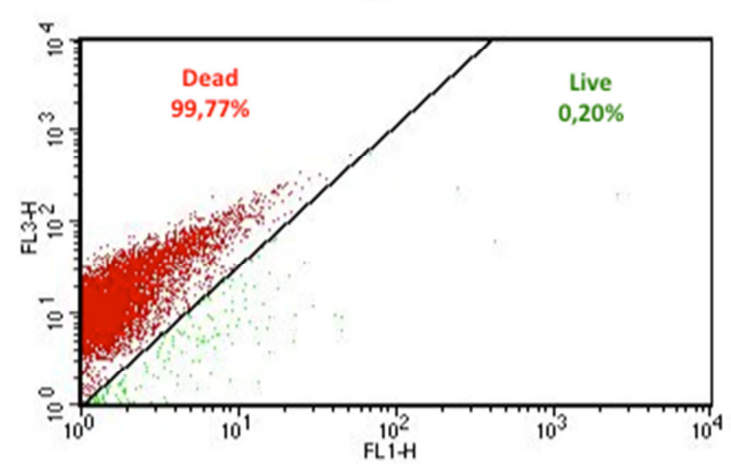

E. coli

(b) Control

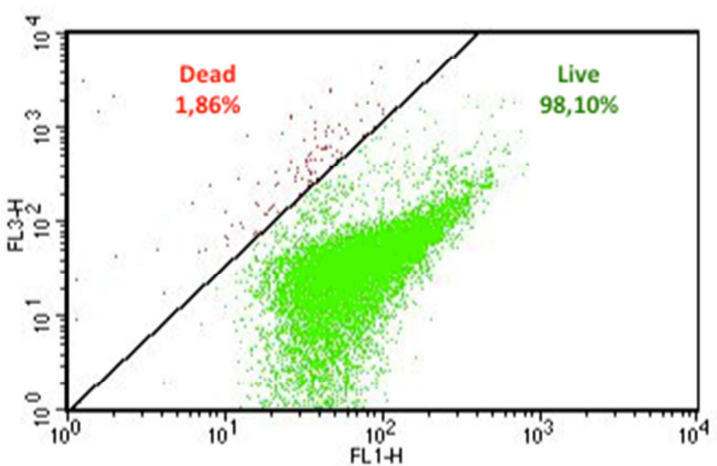

(d)

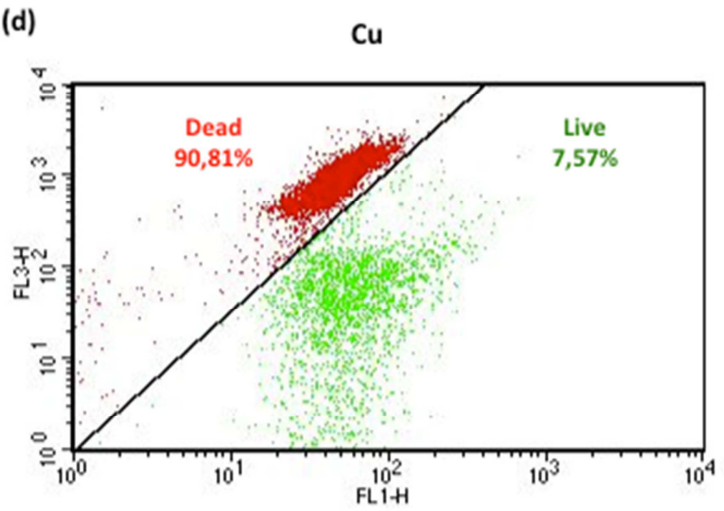

Fig. S3. Flow cytometric dotplot analysis of bacteria stained with the LIVE/DEAD assay. $S$. aureus (a, c) and $E$. coli (b, d) were incubated for $24 \mathrm{~h}$ on the plastic bottom (a, b) or on bare $\mathrm{Cu}(\mathrm{c}, \mathrm{d})$. Each dot of the plot represents a single bacterium which is stained according to its physiological status: red for severe membrane damage (death), green for no detectable membrane damage (presumably alive). Y-axis: red fluorescence with propidium iodide, $\mathrm{X}$ axis: green fluorescence with syto 9 . 


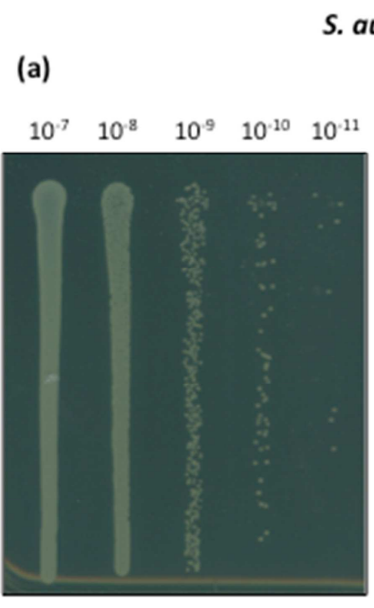

Control
S. aureus

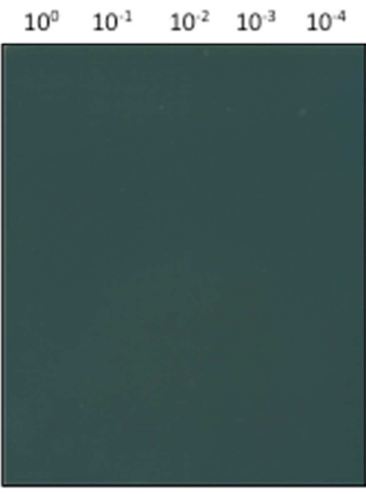

$\mathrm{Cu}$

(b)

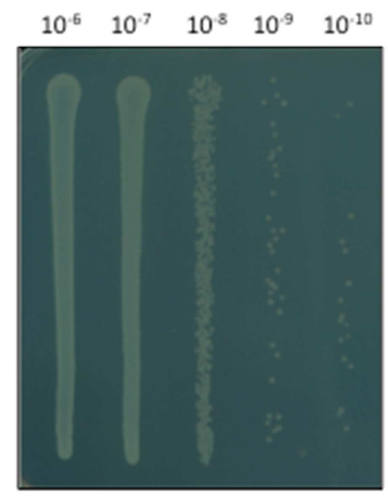

Control
E. coli

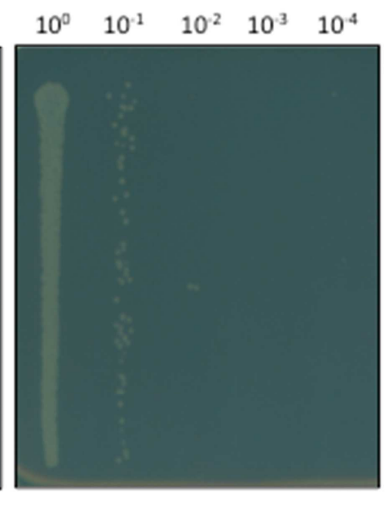

$\mathrm{Cu}$

Fig. S4. Typical photographs of serially diluted S. aureus (a) and E. coli (b) bacteria grown on agar culture plates after a $24 \mathrm{~h}$ incubation on the plastic bottom (control) or on bare $\mathrm{Cu}$. 